\title{
Eicosanoid and amino acid metabolism in transient acute psychoses with psychedelic symptoms
}

\author{
Durk Fekkes ${ }^{1}$, Willem T. Bode', Frederik J. Zijlstra², Lolke \\ Pepplinkhuizen ${ }^{3}$
}

${ }^{1}$ Section Pathophysiology of Behaviour, Erasmus University Rotterdam, Rotterdam, The Netherlands. 2Department of Pharmacology, Erasmus University Rotterdam, Rotterdam, The Netherlands.

${ }^{3}$ Section Pathophysiology of Behaviour/Department of Psychiatry, Erasmus University Rotterdam, Rotterdam, The Netherlands.

\begin{abstract}
Summary It has been hypothesized that a disturbance of glutathione (GSH) metabolism might be a common factor in many psychiatric disorders. The aim of the present study was to test this hypothesis in transient acute psychotic patients with distorted perceptions. Since the metabolism of GSH is related to that of thromboxane $\mathrm{B}_{2}\left(\mathrm{TXB}_{2}\right)$, prostaglandin $E$ (PGE) and some amino acids, we determined these substances in the plasma of 15 patients and 17 normal controls. Plasma concentrations of $\mathrm{TXB}_{2}$ were significantly higher and concentrations of serine and tryptophan were significantly lower in patients than in controls. Large variation was observed in plasma PGE levels in patients, although mean values did not differ significantly from controls. These results are consistent with the hypothesis that the metabolism of GSH is impaired in transient psychotic states.
\end{abstract}

\section{INTRODUCTION}

Eicosanoids are one of the many substances that have been implied in psychosis and schizophrenia. ${ }^{1}$ An early hypothesis that schizophrenia may be a prostaglandin (PG) deficiency disease was based on the following facts: (a) many antipsychotics stimulate prolactin secretion, which in turn stimulates PG synthesis, (b) schizophrenia excludes pain, inflammation and rheumatoid arthritis and concomitant PG levels are low and (c) schizophrenialike syndromes can be induced by PG antagonists. ${ }^{2}$ However, the results of PG supplementation studies are contradictory ${ }^{1}$ and plasma levels of $\mathrm{PGE}_{2}$ in schizophrenic patients were found to be both decreased ${ }^{3}$ and increased. ${ }^{4}$ On the other hand, increased plasma phospholipase $A_{2}$ $\left(\mathrm{PLA}_{2}\right)$ activity has been found in schizophrenic patients

Received 18 January 1995

Accepted 4 April 1995

Correspondence to: Durk Fekkes, Tel. (010) 4087529 or 7545; Fax. (010) 4633217 and antipsychotic drugs like haloperidol, chlorpromazine and trifluoperazine, reduced the activity of PLA $\mathrm{P}_{2} \cdot{ }^{5}$ Since $\mathrm{PLA}_{2}$ is a key enzyme in the formation of arachidonic acid from phospholipids, up-regulation of prostaglandin synthesis in schizophrenia is more likely and the clinical effect of antipsychotic drugs is not due to elevation of prolactin but rather a result of $\mathrm{PLA}_{2}$ inhibition.

A special type of psychosis - the transient acute polymorphic psychosis with or without symptoms of schizophrenia according to ICD-10 criteria ${ }^{6}$ - has been studied by Bruinvels et al ${ }^{7}$ and Fekkes et a1. ${ }^{8}$ This psychosis is characterized by psychosensory symptoms of the type seen after LSD ingestion. From the available biochemical data it was hypothesized that the disturbed metabolism of serine (Ser) in these patients leads to an increased formation of $N^{5}, N^{10}$-methylene-tetrahydrofolate. The latter substance can dissociate into among others formaldehyde, which in turn may react with indolamines yielding $\beta$-carbolines, of which some are hallucinogenic. ${ }^{7}$

Studying the biochemistry of schizophrenic and the above mentioned psychotic patients, it became apparent 
that there is one factor in common with all described biochemical alterations, viz the metabolism of glutathione (GSH). Evidence that alterations in GSH metabolism may be involved in psychiatric manifestations comes from the observation that schizophrenic women exhibit abnormal activities of GSH peroxidase ${ }^{9}$ and that schizophrenics may benefit from GSH infusion..$^{10}$ The hypothesized excess in formaldehyde levels in the transient psychotic patients may be explained by a combination of an increased Ser metabolism and a decreased formaldehyde dehydrogenase activity, due to a low level of its co-enzyme GSH.

In the present study, we examined the alterations in GSH in an indirect way in transient psychotic patients with psychedelic symptoms and normal controls by measuring the plasma levels of some amino acids related to GSH metabolism and the levels of PGE and thromboxane $\mathrm{B}_{2}\left(\mathrm{TXB}_{2}\right)$. The eicosanoids were measured, because the formation of PGE is dependent on the co-factor $\mathrm{GSH}^{11}$ and $\mathrm{TXB}_{2}$ levels have been found to be inversely correlated with GSH levels. ${ }^{12}$

\section{SUBJECTS AND METHODS}

\section{Subjects}

15 transient acute psychotic patients all with distorted sensory perceptions ( 8 female, 7 male; age $=34 \pm 12$ years, mean $\pm \mathrm{SD}$ ), who were drug-free for at least 3 weeks, and 17 normal controls ( 10 female, 7 male; age $=32 \pm 6$ years, mean \pm SD), were included in the study. All patients were diagnosed according to ICD-10 criteria: ${ }^{6} 9$ patients suffered from polymorphic episodic psychosis (F 23.0 and 23.1), 4 from organic delusional schizophrenia-like disorder (F 06.2), 1 from mania with psychotic symptoms ( $F$ 30.2) and 1 from substance abuse disorder (cannabinoids; $F$ 12.53). At the moment of blood sampling the mental states of the patients were rated as not psychotic $(n=3)$, slightly disturbed, e.g. depersonalization $(n=4)$ and fullblown psychotic $(n=8)$. From one polymorphic psychotic patient, in whom psychedelic symptoms could be evoked with Ser, a sample was taken during two different periods of full-blown psychosis. For statistical reasons biochemical determinations of these two samples were averaged.

The study was approved by the Medical Ethical Committee of the university.

\section{Procedures}

Venous blood samples were drawn between 09:00 and 10:00, using $10 \mathrm{ml}$ siliconized monoject tubes containing $0.15 \%$ EDTA. Blood was centrifuged for $20 \mathrm{~min}$ at $20^{\circ} \mathrm{C}$ and $2650 \times g_{\max }$ and the plasma was stored at $-30^{\circ} \mathrm{C}$ until analysis.

\section{Laboratory methods}

Amino acid analysis was performed as described previously. ${ }^{13}$ We also determined the so-called TSM-ratio, which is defined as the ratio of 100 times the taurine (Tau) concentrations in plasma and the product of the plasma concentrations of Ser and methionine (Met). ${ }^{8}$

Determination of the eicosanoids PGE and $\mathrm{TXB}_{2}$ was performed by a simple and routine radioimmunoassay. ${ }^{14}$

\section{Statistics}

Group differences were tested with a multivariate analysis of variance (MANOVA), followed by univariate analysis of variance (ANOVA) for each individual variable. Homogeneity of variance was tested using Box's M-test. The values given are means $\pm \mathrm{SD}$; the level of significance was set at $5 \%$ (two-tailed).

\section{RESULTS}

The plasma concentrations of the measured amino acids and eicosanoids are shown in Table 1 . Ser, Met and PGE were not normally distributed. Therefore, a logarithmic transformation was performed on these variables for statistical analysis. Two cases were omitted due to the occurrence of extreme values. One case was the patient suffering from mania with psychotic symtoms, who exhibited an extremely high plasma level of $\mathrm{TXB}_{2}$, viz $2432 \mathrm{pg} / \mathrm{ml}$ (normal value in this study: $173 \mathrm{pg} / \mathrm{ml}$ ). Although the numerical value was not reliable, this patient was no exception in the way patients deviated from controls. The other case was a patient suffering from polymorphic psychosis, who showed a very low $(18 \mathrm{pg} / \mathrm{ml})$ plasma level of PGE (normal value in this study: $781 \mathrm{pg}$ / $\mathrm{ml}$ ). The multivariate test yielded statistically significant results $(F=4.61 ; \mathrm{df}=7.22 ; P=0.003)$. Univariate $F$-tests showed that this effect could be attributed to differences in Ser, tryptophan (Trp), $\mathrm{TXB}_{2}$ and the TSM-ratio (Table 1).

The plasma Ser and Trp levels of the psychotic patients

Table 1 Concentrations of amino acids, $\mathrm{TXB}_{2}$ and PGE, and TSMratios in plasma of patients and normal controls.

\begin{tabular}{lccl}
\hline Variable & $\begin{array}{l}\text { Controls } \\
(\boldsymbol{n}=\mathbf{1 7})\end{array}$ & $\begin{array}{l}\text { Patients } \\
(\boldsymbol{n}=\mathbf{1 3})\end{array}$ & $\boldsymbol{P}$ value \\
\hline Tau $(\mu \mathrm{mol} / \mathrm{l})$ & $52 \pm 12$ & $51 \pm 11$ & $\mathrm{NS}$ \\
Ser $(\mu \mathrm{mol} / \mathrm{l})$ & $136 \pm 35$ & $107 \pm 21$ & 0.012 \\
Met $(\mu \mathrm{mol} / \mathrm{l})$ & $26 \pm 7$ & $22 \pm 3$ & $\mathrm{NS}$ \\
Trp $(\mu \mathrm{mol} / \mathrm{l})$ & $50 \pm 12$ & $35 \pm 12$ & 0.003 \\
TSM-ratio & $1.69 \pm 0.71$ & $2.30 \pm 0.62$ & 0.020 \\
TXB $(\mathrm{pg} / \mathrm{ml})$ & $173 \pm 106$ & $382 \pm 122$ & $<0.001$ \\
PGE $(\mathrm{pg} / \mathrm{ml})$ & $781 \pm 185$ & $862 \pm 458$ & NS \\
\hline
\end{tabular}

All results are expressed as means $\pm \mathrm{SD} . n=$ number of individuals. NS $=$ not significantly different from controls. 
were significantly lower than those of normal controls (ANOVA, $P=0.012$ and 0.003 , respectively; Table 1). Met levels were also lower, but these differences were not significant. Mean plasma Tau levels did not differ between the two groups. The TSM-ratio appeared to be higher in the patients than in the control group $(P=0.020)$. Of the eicosanoids, the $\mathrm{TXB}_{2}$ levels in plasma of the patients were significantly higher than those of the controls $(P<0.001)$, whereas mean PGE levels did not differ between these groups. It has to be noted that the SD of the PGE levels in plasma of the patients was very high. Even in the same patient, variation in PGE levels were high. For instance, in the plasma of the patient of whom 2 blood samples were taken, the PGE levels were 0 and $1114 \mathrm{pg} /$ $\mathrm{ml}$, respectively.

Further subdivision of patients and controls regarding age, plasma levels of Tau, Ser, Met and Trp, the TSM-ratio, and the plasma levels of $\mathrm{TXB}_{2}$ and $\mathrm{PGE}$ as measured variables against groupings of the subjects in patients and controls with subdivisions in male/female, mental state of the patient and other above mentioned characteristics, did not reveal any further significant differences.

\section{Discussion}

The aim of this study was to find more solid proof for the hypothesis that in a group of psychiatric patients, suffering from transient acute psychotic disorders with psychosensory symptoms, GSH metabolism is altered. Indirect measurement of GSH by way of determination of the plasma levels of the eicosanoids $\mathrm{TXB}_{2}$ and PGE was chosen, because we lack the technique to measure GSH. The two parameters TXB2 and PGE were selected, because $\mathrm{TXB}_{2}$ concentrations have been found to be inversely correlated with GSH concentrations ${ }^{12,15,16}$ and PGE uses GSH as a co-substrate for its formation. ${ }^{11,16}$

As explained above, the patients suffering from polymorphic psychosis are expected to have increased formaldehyde levels. ${ }^{7}$ Whether this is due to a deficiency in GSH synthesis or that excess formaldehyde leads to a reduction in GSH concentration is not clear. In any case, our finding of a two-fold increase in $\mathrm{TXB}_{2}$ in all patients versus controls (Table 1) is in line with a decreased GSH availability. Although the mean plasma PGE levels did not differ between the two groups, its variation in the patient group was 2.5 times higher $(S \mathrm{SD}=494 \mathrm{pg} / \mathrm{ml})$ than in the normal controls $(\mathrm{SD}=185 \mathrm{pg} / \mathrm{ml})$. This indicates that GSH concentrations in episodic psychotic patients may fluctuate rapidly. The patient observed during different periods of psychosis showed during both periods elevated plasma $\mathrm{TXB}_{2}$ levels $\left(708\right.$ and $610 \mathrm{pg} / \mathrm{ml} \mathrm{TXB}{ }_{2}$, respectively), whereas the plasma PGE concentration was at one time

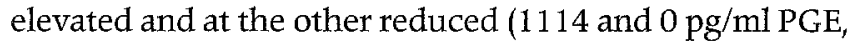
respectively). Probably, the determination of $\mathrm{TXB}_{2}$ tells us more about the direction of change in GSH levels than the measurement of PGE, because the effects of GSH on PGE are somewhat difficult to interpret. For instance, it has been found that formation of $\mathrm{PGE}_{2}$ is inhibited in the presence of $1 \mathrm{mM} \mathrm{GSH}$, whereas $0.1 \mathrm{mM} \mathrm{GSH}$ stimulated its synthesis. ${ }^{17}$ Therefore, $\mathrm{TXB}_{2}$ is regarded as the most important parameter.

A significant decrease in plasma Trp levels was found in the studied psychotic patients. This may be explained by an increase in the synthesis of NADPH - Trp is a precursor of NADPH - in order to keep sufficient GSH in a reduced state. It is conceivable that not only the absolute level of GSH is important, but also the ratio between reduced and oxidized GSH.

The observed increased TSM-ratio in the psychotic patients compared to normal controls is mainly due to a decrease in plasma Ser level, because the levels of Tau and Met were not changed significantly. The plasma Ser levels of the 9 patients suffering from polymorphic psychosis (ICD-10 categories F 23.0 and 23.1) were slightly lower than those of the whole patient group $(98 \pm 21$ and $106 \pm$ $20 \mu \mathrm{mol} / \mathrm{h}$, respectively), while the $\mathrm{TXB}_{2}$ levels were comparable (399 \pm 145 and $373 \pm 122 \mathrm{pg} / \mathrm{ml}$, respectively).

In conclusion, the plasma $\mathrm{TXB}_{2}$ levels of psychotic patients with distorted perceptions were found to be significantly higher than those of normal controls, while the concentrations of the amino acids Trp and Ser were lower. Moreover, the variation in plasma PGE levels of the psychotic patients was more than 2-fold higher than in the control group. All differences found point to a disturbance in the metabolism of GSH in transient acute psychosis and suggest that GSH is involved in the etiology of the psychosensory symptoms. Whether this must be interpreted in terms of active oxygen species involvement or excess formaldehyde formation cannot be answered yet.

\section{ACKNOWLEDGEMENTS}

The authors are grateful to Mr René Verheij for performing the statistical analyses.

\section{REFERENCES}

1. Rotrosen J., Wolkin A. Phospholipid and prostaglandin hypotheses of schizophrenia. In: Meltzer H. Y., ed. Psychopharmacology: The Third Generation of Progress, New York: Raven Press, 1987: 759-764.

2. Horrobin D. F. Schizophrenia as a prostaglandin-deficiency disease. Lancet 1977; 1: 936-937.

3. Mathe A. A., Eberhard G., Sääf J., Wetterberg L. Plasma prostaglandin $\mathrm{E}_{2}$ metabolite - measured as 11-deoxy-15-keto13,14-dihydro-1 $1 \beta, 16 \mathrm{Xi}$-cyclo-PGE $\mathrm{P}_{2}$ - in twins with schizophrenia disorder. Biol Psych 1986; 21: 1024-1030.

4. Idaka E., Kaiya H., Horitsu H. Determination of prostaglandin $\mathrm{E}_{2}$ in plasma of schizophrenics and healthy controls by gas chromatography negative-ion chemical ionisation mass 
spectrometry and radioimmunoassay. J Chromatogr Biomed Appl 1987; 420: 373-378.

5. Gattaz W. F., Köllisch M., Thuren T., Virtanen J. A., Kinnunen P. K. J. Increased plasma phospholipase $\mathrm{A}_{2}$ activity in schizophrenic patients: Reduction after neuroleptic therapy. Biol Psych 1987; 22: 421-426.

6. World Health Organizatlon. ICD-10 Classification of Mental and Behavioural Disorders, Geneva: WHO, 1992.

7. Bruinvels J., Pepplinkhuizen L., van Tuijl H. R., Moleman P., Blom W. Role of serine, glycine, and the tetrahydrofolic acid cycle in schizoaffective psychosis. A hypothesis relating porphyrin biosynthesis and transmethylation. In: Usdin E., Sourkes T. L., Youdim M. B. H., eds. Enzymes and Neurotransmitters in Mental Disease, New York: John Wiley, 1980: 139-154.

8. Fekkes D., Pepplinkhuizen L., Verheij R., Bruinvels J. Abnormal plasma levels of serine, methionine and taurine in transient acute polymorphic psychosis. Psych Res 1994; 51: 11-18.

9. Abdalla D. S., Monteiro H. P., Oliveira J. A., Bechara E. J. Activities of superoxide dismutase and glutathione peroxidase in schizophrenic and manic-depressive patients. Clin Chem 1986; 32: 805-807.

10. Eiduson S., Geller E., Yuwiler A., Eiduson B. T. Attempts at chemical diagnosis of mental aberration. In: Biochemistry and Behavior, Princeton, New Jersey: Van Nostrand 1984: 251-315.
11. Parnham M. J. The pharmacology of antirheumatic drugs Agents Actions 1984; 14: 153-169.

12. Hofmann J., Lösche W., Ostermann G., Till U. Interdependence of GSH and formation of malondialdehyde in arachidonic acid induced platelet activation. In: Förster W., ed. Prostaglandins and Thromboxanes, Proc 3rd Int Symp on Prostaglandins and Thromboxanes in the Cardiovascular System, Halle/Saale, GDR: VEB Gustav Fischer Verlag, 1981: 197-198.

13. Bruinvels J., Pepplinkhuizen L. Impaired glycine-serine conversion and increased plasma taurine levels in episodic psychotic patients with psychedelic symptoms. J Psych Res 1984 18: $307-318$

14. Zijlstra F. J., Vincent J. E., van den Berg B., Hoogsteden H. C., Neyens H. J., van Dongen J. J. M. Pulmonary alveolar proteinosis: Determination of prostaglandins and leukotrienes in lavage fluid. Lung 1987; 165: 79-89.

15. Thomas G., Skrinska V., Lucas F. V., Schumacher O. P. Platelet glutathione and thromboxane synthesis in diabetes. Diabetes 1985; 34: 951-954.

16. Bellan J. A., Minkes R. K., Kerstein M. D. et al. Concentrationactivity profile of the modulation of cyclooxygenase product formation by reduced glutathione in microsomal fractions from the goat lung. Biochim Biophys Acta 1990; 1044: 315-322.

17. Goswami S. K., Gold R. M. Prostanoid synthesis in peripheral nerve. Biochim Biophys Acta 1985; 834: 263-266. 